\title{
Allergic bronchopulmonary aspergillosis in children
}

\author{
Sara Manti ${ }^{1}$, Giuseppe Fabio Parisi ${ }^{2}$, Maria Papale ${ }^{3}$, Amelia Licari ${ }^{4}$, Elena Chiappini ${ }^{5}$, \\ Enza Mulè ${ }^{3}$, Novella Rotolo ${ }^{6}$, and salvatore leonardi ${ }^{3}$ \\ ${ }^{1}$ UOC Genetics and Immunology Paediatrics \\ ${ }^{2}$ University of Catania \\ ${ }^{3}$ University of Catania School of Medicine \\ ${ }^{4}$ Fondazione IRCCS Policlinico San Matteo \\ ${ }^{5}$ University of Florence, Meyer Children's Hospital \\ ${ }^{6}$ University
}

August 11, 2020

\begin{abstract}
Allergic bronchopulmonary aspergillosis (ABPA) is a pulmonary disease caused by Aspergillus induced hypersensitivity that occurs in immunocompetent but susceptible patients with asthma and/or cystic fibrosis (CF). In children, ABPA remains mostly undiagnosed, resulting in one of the most common causes of poorly controlled asthma and highly significant morbidity in children with CF. Currently, no specific diagnostic criteria of ABPA for children are available. Corticosteroids and itraconazole are the mainstays of therapy, althoughthere is a lack of randomized clinical trials regarding their usefulness for ABPA in children. Several monoclonal antibodies, such asomalizumab and mepolizumab, may be potential therapies for refractory ABPA in pediatric patients; however, further data are required to clarify the optimal dose and duration of therapy as a routine treatment approach.
\end{abstract}

\section{Key messages}

In children, allergic bronchopulmonary aspergillosis remains largely undiagnosed resulting in one of the most common causes of poorly controlled asthma and significant morbidity in children with cystic fibrosis.

An early diagnosis allows us to start an earlier treatment of allergic bronchopulmonary aspergillosis that is crucial for preventing the severe sequelae such as impairment in lung function, pulmonary fibrosis, and bronchiectasis.

New, targeted therapies for allergic bronchopulmonary aspergillosis have emerged as effective options complementing the understanding of host-Aspergillus interaction.

Firstly described in the United Kingdom in 1952, Allergic bronchopulmonary aspergillosis (ABPA) is a pulmonary disease caused by Aspergillus, principally Aspergillus fumigatus, induced hypersensitivity that occurs in immunocompetent but susceptible patients with asthma and/or cystic fibrosis (CF) [1]. The prevalence of ABPA in the pediatric population is not known. The first pediatric case of ABPA was reported in 1970, since then only rare case reports and small case series have been described. ABPA may be found in up to $6 \%$ of all asthmatic patients, and from $2 \%$ to $15 \%$ in CF patients [1]. Age more than 12 years, low-CF index, presence of immunoglobulin (Ig)E and eosinophilia, bronchial colonization with Stenotrophomonas maltophilia and Pseudomonas aeruginosa, long-term azithromycin therapy, and higher cumulative doses of inhaled corticosteroids (CS) have been reported as risk factors for ABPA in CF children [2]. 
At present, the underlying pathophysiology of ABPA is not clearly understood. Repeated inhalation of Aspergillus spores causes airway colonization, the release of proteolytic enzymes and pro-inflammatory cytokines, and, lastly, evokes a T-helper (Th) cell -2 type immune response as well as a polyclonal antibody response leading to increased serum IgE-, IgG- and IgA antibodies levels [3]. An impaired balance between human leukocyte antigen (HLA)-antigen D-related molecules associated with susceptibility and resistance to ABPA has been hypothesized to be also involved in the pathogenesis of ABPA [3].

Clinically, children with ABPA may present both systemic symptoms such as fever and weight loss, and respiratory symptoms, including poorly controlled asthma, wheezing, increased productive cough, tachypnea/dyspnea, chest pain, hemoptysis, and exacerbations responding poorly to antibiotics. The reported mean age on clinical presentation is $12.9 \pm 4$ years as a latent period up to 10 years before diagnosis had been described and male preponderance has been also reported [2]. At physical examination crackles and rhonchi are usually reported. Patients with ABPA show a severe progression in all lung function parameters, and, $\mathrm{FEF}$ at $50 \% \mathrm{VC}\left(\mathrm{FEF}_{50}\right)$, the volume of trapped gas, and specific airway resistance as the best predictors have been reported $[1,2,4]$. Recently, exhaled nitric oxide $\left(\mathrm{FE}_{\mathrm{NO}}\right)$ has been proposed as biomarker for the diagnosis of ABPA in $\mathrm{CF}[5,6]$. It has been assessed that an early disease recognition may prevent the progression of ABPA, and, accordingly, clinical and radiographic stages of ABPA progression have been proposed $[7,8]$. The radiographic findings of ABPA on chest-X-ray are not specific, but pulmonary infiltrates, consolidation, and macronodular densities are commonly reported at the early-stage disease, whereas, central bronchiectasis, ring shadows, and pulmonary fibrosis are reported at the end-stage. Chest computed tomography (CT) demonstrates high attenuation mucus plugs and different patterns of central, cylindrical, varicose, and cystic bronchiectasis, that, involving multiple bronchi, appear described as "a string of pearls" or "signet ring sign" [7, 8]. Recently, magnetic resonance imaging (MRI) has also been proposed as a diagnostic tool in ABPA since it appears highly specific (100\%) and sensitive (94\%) for the diagnosis of ABPA in $\mathrm{CF}$, detecting the inverted mucoid impaction signal, defined by the presence of mucus with both high T1 and low T2 signal intensities [7,8]. The diagnostic criteria for ABPA in children are the same used in the adult population. In addition to the presence of a predisposing condition such as asthma or CF, patient must show increased IgE against Aspergillus fumigatus $(>0.35 \mathrm{kUA} / \mathrm{L})$, and total serum IgE $>1000 \mathrm{IU} / \mathrm{ml}(2400$ $\mathrm{ng} / \mathrm{mL}$ ), and, at least two out of three secondary criteria must be satisfied including radiographic transient or fixed findings consistent with ABPA; serum IgG $>27 \mathrm{mg} / \mathrm{L}$ against Aspergillus fumigatus ; and elevated serum eosinophils levels (>500 mmc) [7]. Because of the clinical (pulmonary infiltrate, bronchiectasis, exacerbations) and radiographic (central bronchiectasis) findings are similar between ABPA and CF, the diagnosis of ABPA may be difficult in this cluster of patients. Therefore, the CF Foundation Consensus has suggested the diagnostic criteria and criteria for screening for ABPA in CF patients [3]. Detection of acute/subacute clinical deterioration not related to other etiologies, serum total $\mathrm{IgE}$ concentration major than $1000 \mathrm{IU} / \mathrm{mL}$ (or $2400 \mathrm{ng} / \mathrm{mL}$ ), cutaneous reactivity to Aspergillus fumigatus, or presence of specific IgE to Aspergillus fumigatus, precipitating antibodies toAspergillus fumigatus, and new or recent abnormalities on imaging investigations are defining a patient with CF affected by ABPA [3]. The goals of the treatment of ABPA are to eradicate colonization and/or proliferation of Aspergillus fumigatus ; suppress inflammatory response; reduce pulmonary exacerbation and prevent fibrotic end-stage disease. The most effective treatment for the acute phase of ABPA is prednisolone at the recommended dosage is $0.5 \mathrm{mg} / \mathrm{kg} /$ day for the first 2 weeks, followed by a progressive tapering over the next $12-16$ weeks in patients with asthma [7]; $0.5-2.0 \mathrm{mg} / \mathrm{kg} /$ day (maximum $60 \mathrm{mg}$ ) for 1-2 weeks, then $0.5-2.0 \mathrm{mg} / \mathrm{kg} /$ day every other day for 1-2 weeks, and then taper in next 2-3 months in patients with CF $[3,7]$. There are several doubts on the efficacy of itraconazole in children with ABPA, since the available literature data are mainly based on data generated in adults. Thus, it is not currently recommended as first-line treatment. The recommended dose of itraconazole is $5 \mathrm{mg} / \mathrm{kg} / \mathrm{day}$, a maximum $400 \mathrm{mg} /$ day (in two divided doses if the total daily dose exceeds $200 \mathrm{mg}$ ) and 3-6 months of a total duration of therapy is suggested [7]. The evidence of a slow or poor response to CS, relapse of the disease in CS-dependent ABPA, and CS-induced toxicity suggest the use of itraconazole in treating of ABPA in patients with $\mathrm{CF}[3,7]$. The rationale for the use of omalizumab, a humanized monoclonal anti-IgE, in the ABPA, is due to its ability to prevent allergen-induced IgE-mediated. This therapy is recommended to be administered as a subcutaneous injection. The dose and frequency of dosing are guided by a nomo- 
gram derived from total serum IgE level and body weight in kilograms. Patients with ABPA and treated with omalizumab reported a significant reduction in exacerbation rates, oral CS exposure, and CS toxicity [1-3, 9]. Recently, mepolizumab, a monoclonal antibody against interleukin (IL)-5 has been proposed as an alternative treatment in ABPA [10]. Other adjuvant therapies such as amphotericin B, voriconazole, isavuconazole, and vitamin D have been proposed in treating ABPA, but further studies are required to assess their efficacy [3]. The monitoring treatment response in ABPA should include the determination of total serum IgE levels every 3 to 6 months/year since it has been suggested that a decrease in total $\operatorname{IgE}$ levels, at least $25 \%$ from baseline, is associated with a clinical improvement, on the contrary, an increase by $>50 \%$ is associated with pulmonary exacerbations [3]. Spirometry should be performed every 3 to 6 months because of a decline of $15 \%$ in functional vital capacity (FVC), which may be a warning signal of ABPA exacerbation [3]. The re-evaluation of environmental exposure and chest-X-ray for patients with $\operatorname{IgE}$ level double, even if asymptomatic, should also be performed. A synacthen test is suggested when adrenal insufficiency is suspected [3].

In summary, many advances in the understanding of pathophysiology have been made and several treatment options for ABPA are currently available. However, whereas in the adult population ABPA is a wellrecognized entity, in children, it remains mostly undiagnosed, resulting in one of the most common causes of poorly controlled asthma and highly significant morbidity in children with CF. An early diagnosis allows us to start an earlier treatment of ABPA that is crucial for preventing the severe sequelae such as impairment in lung function, pulmonary fibrosis, and bronchiectasis.

\section{References}

1. Hinson KF, Moon AJ, Plummer NS. Broncho-pulmonary aspergillosis; a review and a report of eight new cases. Thorax 1952;7(4):317-333

2. Sharma VK, Raj D, Xess I, Lodha R, Kabra SK. Prevalence and risk factors for allergic bronchopulmonary aspergillosis in Indian children with cystic fibrosis. Indian Pediatr 2014;51:295-7

3. Stevens DA, Moss RB, Kurup VP, Knutsen AP, Greenberger P, Judson MA, Denning DW, Crameri R, Brody AS, Light M, Skov M, Maish W, Mastella G; Participants in the Cystic Fibrosis Foundation Consensus Conference. Allergic bronchopulmonary aspergillosis in cystic fibrosis - State of the art: Cystic fibrosis foundation consensus conference. Clin Infect Dis 2003;37 Suppl 3:S225-64

4. Kraemer R, Delosea N, Ballinari P, Gallati S, Crameri R. Effect of allergic bronchopulmonary aspergillosis on lung function in children with cystic fibrosis. Am J Respir Crit Care Med 2006;174(11):1211-1220

5. Keown K, Abbott S, Kuzeljevic B, Rayment JH, Chilvers MA, Yang CL. An investigation into biomarkers for the diagnosis of ABPA and aspergillus disease in cystic fibrosis. Pediatr Pulmonol. 2019;54(11):1787-1793

6. Leonardi S, Cuppari C, Lanzafame A, Attardo D, Tardino L, Parisi G, Giacchi V, Manti S, Arrigo T. Exhaled breath temperature in asthmatic children. J Biol Regul Homeost Agents. 2015;29(2 Suppl 1):47-54.

7. Patterson R, Greenberger PA, Radin RC, Roberts M. Allergic bronchopulmonary aspergillosis: Staging as an aid to management. Ann Intern Med 1982;96:286-91

8. Agarwal R, Sehgal IS, Dhooria S, Aggarwal AN. Developments in the diagnosis and treatment of allergic bronchopulmonary aspergillosis. Expert Rev Respir Med 2016;10:1317-34

9. Parisi GF, Portale A, Papale M, Tardino L, Rotolo N, Licari A, Leonardi S. Successful treatment with omalizumab of allergic bronchopulmonary aspergillosis in patients with cystic fibrosis: Case reports and literature review. J Allergy Clin Immunol Pract. 2019;7(5):1636-1638

10. Hirota S, Kobayashi Y, Ishiguro T, Nishida T, Kagiyama N, Shimizu Y, Takayanagi N. Allergic bronchopulmonary aspergillosis successfully treated with mepolizumab: Case report and review of the literature. Respir Med Case Rep 2018;26:59-62. 